\title{
CrystEngComm
}

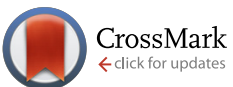

Cite this: CrystEngComm, 2016, 18, 7722

Received 29th July 2016,

Accepted 30th August 2016

DOI: $10.1039 /$ c6ce01671e

www.rsc.org/crystengcomm

\section{A microfluidic-based protein crystallization method in 10 micrometer-sized crystallization space $\uparrow$}

\author{
Masatoshi Maeki, ${ }^{\star a b}$ Shohei Yamazaki, ${ }^{c}$ Ashtamurthy S. Pawate, ${ }^{d}$ Akihiko Ishida, ${ }^{a}$ \\ Hirofumi Tani, ${ }^{a}$ Kenichi Yamashita, ${ }^{b}$ Masakazu Sugishima, ${ }^{e}$ Keiichi Watanabe, ${ }^{f}$ \\ Manabu Tokeshi, ${ }^{a}$ Paul J. A. Kenis ${ }^{d}$ and Masaya Miyazaki*b
}

\begin{abstract}
Protein crystallization and subsequent X-ray diffraction analysis of the three-dimensional structure are necessary for elucidation of the biological functions of proteins and effective rational drug design. Therefore, controlling protein crystallization is important to obtain high resolution X-ray diffraction data. Here, a simple microfluidic method using chips with 10 and $50 \mu \mathrm{m}$ high crystallization chambers to selectively form suitable single protein crystals for X-ray analysis is demonstrated. As proof of concept, three different types of proteins: lysozyme, glucokinase from Pseudoalteromonas sp. AS-131 (PsGK), and NADPH-cytochrome P450 oxidoreductase-heme oxygenase complex were crystallized. We demonstrate that the crystal growth orientation depends on the height of the crystallization chamber regardless of the protein type. Our results suggest that the confined micro space induces the protein molecules to adhere to a specific crystal face and affects the growth kinetics of each crystal face. The present microfluidic-based protein crystallization method can reform a suitable single protein crystal for X-ray analysis from aggregates of needle-shaped protein crystals.
\end{abstract}

\section{Introduction}

A combination of structure-based drug design (SBDD) and high-throughput screening offers effective development of new drugs and can minimize the development costs, time, and labor. ${ }^{1,2}$ The computational modeling of a protein structure for SBDD requires correct and precise information about the three-dimensional structure of the target proteins. Therefore, high-resolution X-ray crystal structure analysis using high quality protein crystals is indispensable for protein structure determination. However, producing protein crystals

\footnotetext{
${ }^{a}$ Division of Applied Chemistry, Faculty of Engineering, Hokkaido University, Kita 13 Nishi 8, Kita-ku, Sapporo 060-8628, Japan.

E-mail:m.maeki@eng.hokudai.ac.jp

${ }^{b}$ Advanced Manufacturing Research Institute, National Institute of Advanced Industrial Science and Technology (AIST), 807-1 Shuku, Tosu 841-0052, Japan. E-mail:m.miyazaki@aist.go.jp

${ }^{c}$ Graduate School of Chemical Sciences and Engineering, Hokkaido University, Kita 13 Nishi 8, Kita-ku, Sapporo 060-8628, Japan

${ }^{d}$ Department of Chemical and Biomolecular Engineering, University of Illinois at Urbana-Champaign, 600 South Mathews Avenue, Urbana, IL 61801, USA

${ }^{e}$ Department of Medical Biochemistry, Kurume University School of Medicine, 67 Asahi-machi, Kurume 830-0011, Japan

${ }^{f}$ Department of Applied Biological Sciences, Saga University, 1 Honjo, Saga 8408502, Japan

$\dagger$ Electronic supplementary information (ESI) available. See DOI: 10.1039/ c6ce01671e
}

of high diffraction quality depends strongly on the skills of the persons growing the crystals and requires a trial and error process. For the production of high quality protein single crystals, large amounts of protein samples and crystallization reagents need to be prepared in order to carry out many crystallization trials. This is a serious drawback in the protein 3D structure determination process, even though suitable protein crystallization conditions might be found during an initial screen. The aggregation of protein crystals must be prevented for X-ray diffraction measurement, since an aggregated protein crystal makes analysis of the protein 3D structure difficult and lowers the resolution limit. For these reasons, development of a method to form a protein single crystal suitable for X-ray analysis is strongly desired.

In the last decade, a variety of techniques and methods have been developed to facilitate protein crystallization experiments. $^{3-5}$ Microfluidics-based platforms offer promising approaches for the screening of protein crystallization conditions and in situ X-ray analysis. ${ }^{6-11}$ A microfluidic chip is able to reduce the consumption of protein samples and crystallization reagents because the required volume of one protein crystallization trial is a few nanoliters to a few tens of nanoliters. In addition, in situ X-ray analysis allows for a simple measurement process compared with conventional manual handling of specimens for X-ray analysis. Recently, several in situ X-ray analysis methods have been reported that 
improve the efficiency of diffraction data collection and can be applied to membrane protein crystallography studies. ${ }^{12-14}$ We have also demonstrated the potential for droplet-based microfluidics to obtain a protein single crystal. ${ }^{15,16}$ We found that the droplet size was an essential parameter for controlling protein crystallization. A few hundred micrometer-sized droplets achieved a diffusion-controlled protein crystallization environment and isolated protein crystals formed in each microdroplet. A precisely controlled space has a potential for generating a single protein crystal with high diffraction quality. However, the effects of a confined crystallization space smaller than $50 \mu \mathrm{m}$ are not well understood.

Herein, we report a simple method using microfluidic chips with 10-50 $\mu \mathrm{m}$ deep crystallization chambers to form suitable protein crystals for X-ray protein crystallography. Three different types of proteins - a typical model protein, a cold adaptation enzyme, and a heme oxygenase complex were chosen for proof-of-concept experiments. Protein crystal growth behavior in the confined micro space was also investigated that led to the elucidation of a novel crystal growth phenomenon regardless of the types of proteins.

\section{Experimental}

\section{Protein solutions and protein crystallization conditions}

Hen egg white lysozyme was purchased from Hampton Research (Aliso Viejo, CA, USA). Glucokinase from Pseudoalteromonas sp. AS-131 (PsGK) and NADPH-cytochrome P450 oxidoreductase-heme oxygenase complex (CPR-HO complex) were prepared using an $E$. coli expression system and purified by column chromatography as previously described. ${ }^{17,18}$ Lysozyme was dissolved at $80 \mathrm{mg} \mathrm{mL}^{-1}$ in $100 \mathrm{mM}$ sodium acetate buffer (pH 4.5; Wako Pure Chemicals, Osaka, Japan). PsGK was dissolved at $7.3 \mathrm{mg} \mathrm{mL} \mathrm{mL}^{-1}$ in $20 \mathrm{mM}$ Tris-HCl buffer $(\mathrm{pH}$ 7.6), and $10 \mathrm{mM} \mathrm{MgCl}_{2}$. CPR-HO complex was prepared in $20 \mathrm{mM}$ Tris-HCl buffer ( $\mathrm{pH}$ 7.4) and was concentrated to 40 $\mathrm{mg} \mathrm{mL}^{-1}$ by ultrafiltration. Microbatch and hanging drop vapor diffusion methods were employed for the batchwise protein crystallization experiments. All solutions were filtered through a $0.20 \mu \mathrm{m}$ syringe filter (Minisart RC4 or RC25, Sartorius Stedim Biotech, Gottingen, Germany) prior to use in the protein crystallization experiments. For microbatch crystallization of lysozyme, an equal volume of lysozyme and the precipitant solution $(1.4 \mathrm{M} \mathrm{NaCl}$ in $100 \mathrm{mM}$ sodium acetate buffer pH 4.5) were mixed in a microtube. $2 \mu \mathrm{L}$ aliquots of this mixture were pipetted into a 96 well microtiter plate and each filled well was covered with mineral oil (M8410, SigmaAldrich). The wells were then sealed with Crystal Clear Sealing Tape (HR3-511, Hampton Research) and the plate was stored in an incubator at $20^{\circ} \mathrm{C}$. PsGK and CPR-HO complex were crystallized by hanging drop vapor diffusion method. For PsGK crystallization, Index 62 from Hampton Research (0.2 M trimethylamine $N$-oxide dihydrate, $0.1 \mathrm{M}$ Tris-HCl (pH 8.5), and 20\% (w/v) PEG MME 2000) was used as a precipitant solution. CPR-HO complex was crystallized using a mixture of $18 \%$ (w/v) PEG 6000, $0.2 \mathrm{M}$ sodium ace- tate, and $0.1 \mathrm{M}$ Tris-HCl ( $\mathrm{pH} 8.6)$ as a precipitant solution. Hanging drop crystallization (24-well VDX plate, Hampton Research) was performed by pipetting the precipitant solution into the reservoir, following which $1 \mu \mathrm{L}$ of protein solution and $1 \mu \mathrm{L}$ of precipitant solution were mixed onto a siliconized glass cover slide (HR3-231, Hampton Research) and the plate was then stored in an incubator at $4{ }^{\circ} \mathrm{C}$ and $20{ }^{\circ} \mathrm{C}$ for PsGK and CPR-HO complex, respectively. Lysozyme crystals formed after incubation for several hours. Crystals of PsGK and CPR-HO appeared after incubation for a few weeks.

For microfluidic chip-based protein crystallization, we employed two different crystallization techniques, a typical free-interface diffusion technique and a microseeding technique for the lysozyme crystallization and for the crystallization of PsGK and CPR-HO complex, respectively. Fig. 1 shows a photograph of the chip and a schematic illustration of the chip used in the protein crystallization experiments. Microseeding on chip was implemented as described previously. ${ }^{19}$ For the microseeding technique, the seed crystals of both proteins were prepared by a hanging drop vapor diffusion method. A schematic illustration of the experimental procedure is shown in Fig. 2. Briefly, the crystallization solution (a mixture of the protein and precipitant solutions) and the seed solution (for the crystallization of PsGK and a complex of CPR-HO; for the lysozyme crystallization, no seed solution was used) were pipetted to the microfluidic chip and introduced into the crystallization chamber by using a vacuum pump. These solutions were mixed by free interface diffusion via a normally closed valve for 5,15 , or $30 \mathrm{~min}$. Each crystallization chamber is $0.67 \mathrm{~mm}$ wide, $0.75 \mathrm{~mm}$ high, and 10 or $50 \mu \mathrm{m}$ deep. The volume of crystallization solution was calculated to be $5 \mathrm{~nL}$ and $25 \mathrm{~nL}$ for $10 \mu \mathrm{m}$ deep chip and $50 \mu \mathrm{m}$ deep chip, respectively. Lysozyme and CPR-HO crystallization experiments were carried out at $20^{\circ} \mathrm{C}$ and the PsGK crystallization experiment was carried out at $4^{\circ} \mathrm{C}$.

A PsGK crystal was evaluated using an optical microscope (ECLIPSE Ti-U, Nikon, Tokyo, Japan) and X-ray diffraction
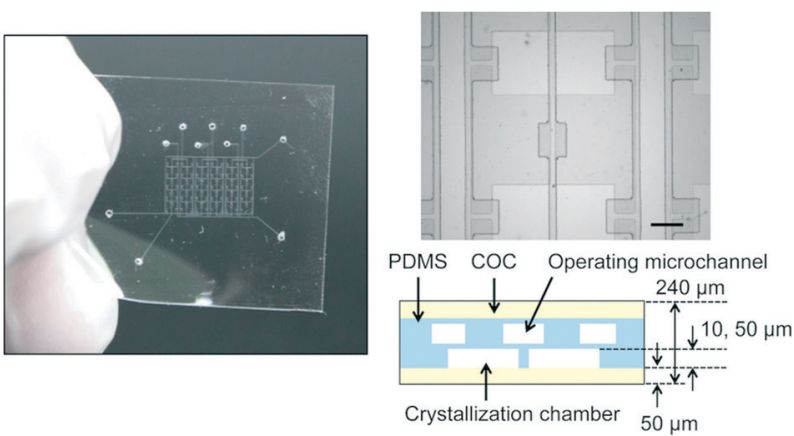

Fig. 1 Photographs and cross-sectional view of the microfluidic chip. The microfluidic chip has 24 crystallization chambers. Each crystallization chamber is $0.67 \mathrm{~mm}$ wide, $0.75 \mathrm{~mm}$ high, and 10 or $50 \mu \mathrm{m}$ deep. The scale bar represents $300 \mu \mathrm{m}$. Protein solutions are introduced to the crystallization chambers by a vacuum pump that reduced the pressure of the operating microchannel. 
(1) Injection

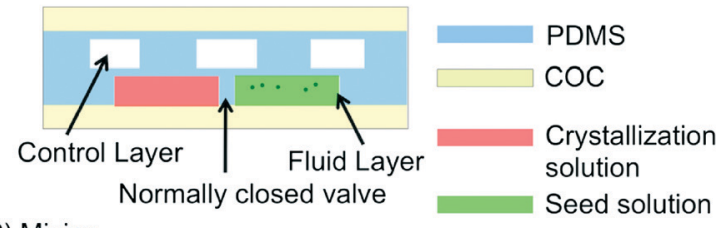

(2) Mixing

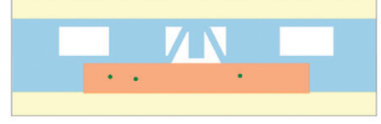

(3) Crystallization

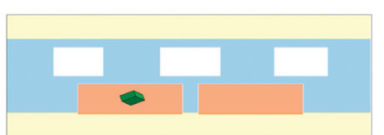

Fig. 2 Protein crystallization experiment procedure using the microfluidic chip. (1) The crystallization solution and the seed solution were introduced to the crystallization chamber using a vacuum pump. (2) The crystallization solution and the seed solution were mixed by counter diffusion via the mixing valve. (3) Inlets of the microfluidic chip were sealed with crystal clear sealing tape. The microfluidic chip was stored in an incubator.

measurement. Lysozyme and CPR-HO complex crystals were observed using the optical microscope.

\section{Fabrication of the microfluidic chip}

Microfluidic chips were fabricated by standard soft lithography with minor modifications. ${ }^{20}$ Polydimethylsiloxane (PDMS; SILPOT 184 W/C, Dow Corning Toray, Tokyo, Japan) and a film of cyclic olefin copolymer (COC; TOPAS 6013, 50 $\mu \mathrm{m}$ thickness, Polyplastics Co., Ltd., Tokyo, Japan) were used as the chip material. First, SU-8 masters for the fluid layer (FL) and control layer (CL) were prepared using epoxy-based photoresist SU-8 3050 (Nippon Kayaku Co., Ltd., Tokyo, Japan) spin-coated (50 $\mu \mathrm{m}$ and $10 \mu \mathrm{m}$ thickness) onto silicon wafers and exposed to UV light (M-1S, Mikasa Co., Ltd, Tokyo, Japan) through appropriate photomasks. The masters were silanized by treatment with a vapor of trichloro(1H,1H,2H,2H-perfluorooctyl)silane (Sigma-Aldrich). The FL (crystallization chamber, 10 or $50 \mu \mathrm{m}$ height) and CL (operating microchannel, $50 \mu \mathrm{m}$ height) masters were spincoated with PDMS to a thickness of $70 \mu \mathrm{m}$. The CL was bonded to a $50 \mu \mathrm{m}$ thick COC film substrate using an oxygen plasma (CUTE-1MP/R, Femto Science, Gwangju, Korea) and the assembly was peeled from the SU-8 master. Then, the assembly was aligned to the FL and baked for at least $4 \mathrm{~h}$ at $80{ }^{\circ} \mathrm{C}$.

\section{On-chip X-ray diffraction measurement}

A PsGK crystal was analyzed by on-chip X-ray diffraction measurement on a synchrotron source (BL 07 at the SAGA Light Source, Saga, Japan). The on-chip X-ray diffraction measurement was made at a wavelength of $1.5 \AA$, with $30 \mathrm{~s}$ exposure and $1^{\circ}$ oscillation and at 277 K. 90 diffraction images were collected. Detailed experimental procedures were reported previously. ${ }^{12}$ Diffraction data were analyzed using XDS software and the CCP4 suite programs. Molecular replacement for PsGK was carried out using PDB 3VPZ data as the model.

\section{Results and discussion}

We selected polydimethylsiloxane (PDMS) and a cyclic olefin copolymer (COC) as chip material because these polymers are easily fabricated into microfluidic chips and exhibit good optical and X-ray transmission properties. ${ }^{21}$ To confirm the effects of the confined space on protein crystallization, two types of microfluidic chips with 10 and $50 \mu \mathrm{m}$ deep crystallization chambers were fabricated. We used lysozyme, glucokinase from Pseudoalteromonas sp. AS-131 (PsGK), and NADPHcytochrome $\mathrm{P} 450$ oxidoreductase-heme oxygenase complex (CPR-HO complex) as model proteins.

Fig. 3 shows photographs of protein crystals formed by batchwise methods and the microfluidic chip method. In the case of batchwise methods, a number of protein crystals formed in the crystallization droplet $(2 \mu \mathrm{L})$ regardless of the type of proteins. In particular, PsGK and CPR-HO complex crystals were significantly aggregated, as shown in Fig. 3(b and c). These crystals were not suitable for X-ray crystal structure analysis because the aggregations lower the diffraction quality. On the other hand, single protein crystals formed within the $50 \mu \mathrm{m}$ deep crystallization chambers in the microfluidic chips, as shown in Fig. 3(d-f). For the crystallization of PsGK and CPR-HO complex, the microseeding technique was employed to control the protein crystallization. The appropriate seeding conditions for PsGK were explored to determine the best concentration of the seed solution, the mixing time between the crystallization solution and the seed solution, and the mixing solution ratio. Photos of crystals grown under different conditions are reproduced in Fig. S1 and S2 in the ESI. $\uparrow$ The optimal protein crystal for X-ray (a)

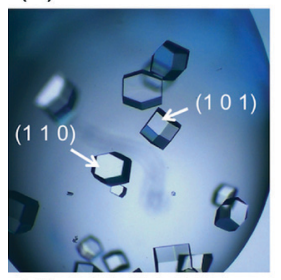

(d)

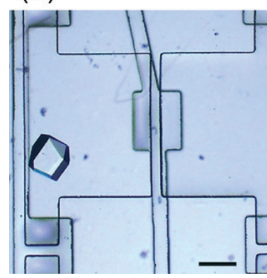

(b)

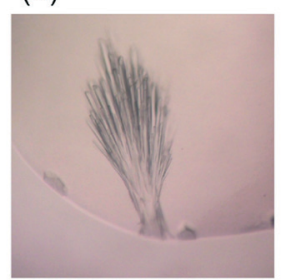

(e) (c)

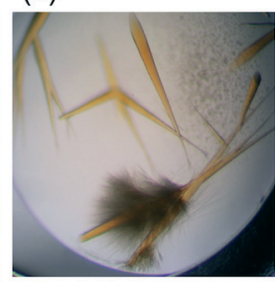

(f)

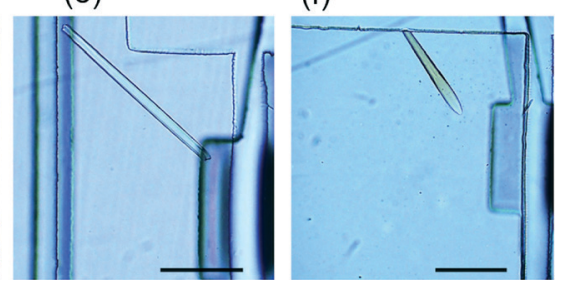

Fig. 3 Photographs of protein crystals formed by the (a) microbatch method, (b, c) hanging drop vapor diffusion method, and the (d-f) microfluidic chip method. (a, d) Lysozyme crystal, (b, e) PsGK crystal, and (c, f) CPR-HO complex crystal. The scale bars represent $200 \mu \mathrm{m}$. 
analysis could be formed with just the valve operation of the microfluidic chip after an incubation of 2-3 weeks. Rodshaped PsGK and CPR-HO crystals formed in each crystallization chamber, and the crystal shapes of these proteins were not different from those obtained by the hanging drop vapor diffusion method.

We observed interesting protein crystallization behavior in the confined crystallization space. Fig. 4 shows the protein crystals formed in the $10 \mu \mathrm{m}$ depth microfluidic chip. In the case of lysozyme crystallization, the (110) face was mainly oriented parallel to the substrate, as shown in Fig. 4(a and b). In contrast, lysozyme crystals from the microbatch method and the $50 \mu \mathrm{m}$ depth microfluidic chip method did not show preference for any specific orientation of crystal growth. As shown in Fig. 4(c), the percentage of the (110) faces grown in the $10 \mu \mathrm{m}$ depth microfluidic chip was almost $100 \%$, which was twice that in the $50 \mu \mathrm{m}$ depth microfluidic chip. Furthermore, the growth direction of lysozyme crystals also differed from the typical crystallization direction, as shown in Fig. 4(b). Seventy percent of the lysozyme crystals grew along the $M$ axis in the $10 \mu \mathrm{m}$ depth microfluidic chip. According to the literature, the $M$ axis grows prior to the $L_{A}$ axis under low supersaturation conditions. ${ }^{22}$ These results indicate that the size of the crystallization space affected the protein crystal growth. In other words, the confined crystallization space promoted the adsorption of protein molecules to a specific crystal face and changed the growth kinetics of each crystal face. We assume that the concentration gradient formed around the crystal after nucleation and crystal growth at an early stage, and consequently, a diffusion-controlled crystallization environment like that of a microgravity environment was achieved by the confined crystallization space due to the suppression of natural convection. ${ }^{23,24}$

The growth time course of lysozyme crystals in the $10 \mu \mathrm{m}$ depth microfluidic chip is shown in Fig. S3. $\dagger$ After a $1 \mathrm{~h} \mathrm{incu-}$ bation, the (110) crystal was not oriented to the substrate; however, we eventually obtained the (110) crystal oriented parallel to the substrate. This suggests that the shape of the
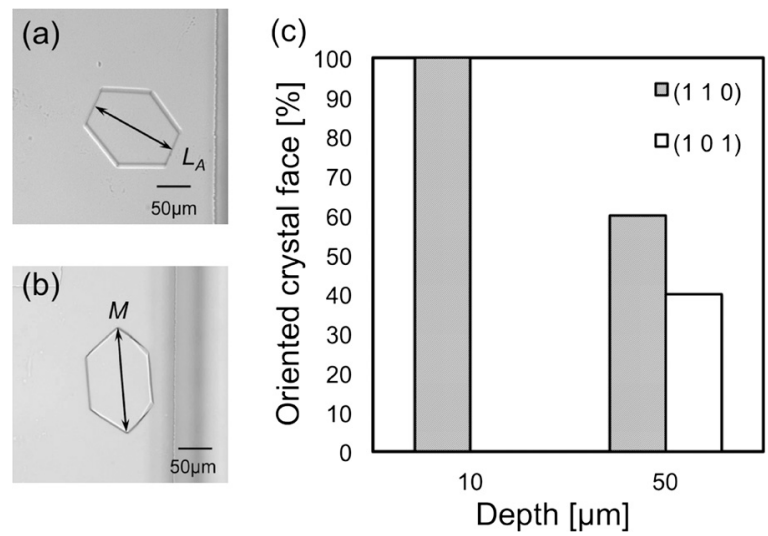

Fig. 4 (a, b) Photographs of the lysozyme crystals formed in the microfluidic chip with $10 \mu \mathrm{m}$ deep crystallization chambers. (c) Percentage of the crystal faces oriented parallel to the substrate. We observed 100-150 crystallization chambers. crystal and the oriented crystal face did not dominate at the nucleation stage. To confirm the site-specific protein molecular adsorption on the crystal face and the potential of the microfluidic-based crystallization approach for generating high quality protein crystals, we investigated PsGK crystallization using the $10 \mu \mathrm{m}$ depth microfluidic chip. Plate-shaped PsGK crystals formed in the microfluidic chip and the crystal shape was dramatically different compared with that of the $50 \mu \mathrm{m}$ depth microfluidic chip (Fig. 5(a and b)). As shown in Fig. 5(c), the plate-shaped PsGK crystals represented almost $60 \%$ of the crystals formed in the $10 \mu \mathrm{m}$ depth microfluidic chip. In contrast, the plate-shaped crystals were seldom observed in the $50 \mu \mathrm{m}$ depth microfluidic chip, and mainly rodshaped PsGK crystals formed in the chambers. The percentage of plate-shaped crystals depended only on the crystallization space, and the crystallization conditions (mixing time, dilution ratio of the seed solutions and mixing ratio) did not affect the shape of the PsGK crystals.

The plate-shaped PsGK crystals were analyzed by in situ X-ray diffraction measurement on a synchrotron radiation source at a wavelength of $1.5 \AA$ at $277 \mathrm{~K}$. Table 1 shows the crystallographic data of the PsGK crystals grown using the hanging drop vapor diffusion method and the microfluidic chip method. The PsGK crystals diffracted to a resolution limit of $2.8 \AA$ without cryoprotection, which is the pretreatment for X-ray diffraction measurement. The space group for the crystal formed by the microfluidic chip method was determined as $P 3_{1} 21$, which is the same as that reported for the crystal formed by the hanging drop vapor diffusion method. The unit cell of the PsGK crystal formed by the chip method is larger than that of the PsGK crystal formed by hanging drop vapor diffusion. ${ }^{14}$ Typically, protein crystals contain many water molecules in the unit cell. Hence, the unit cell of a protein crystal decreases because of rapid dehydration that occurs with cryoprotection. In the present study, we did not use cryoprotection of the PsGK crystal; therefore,
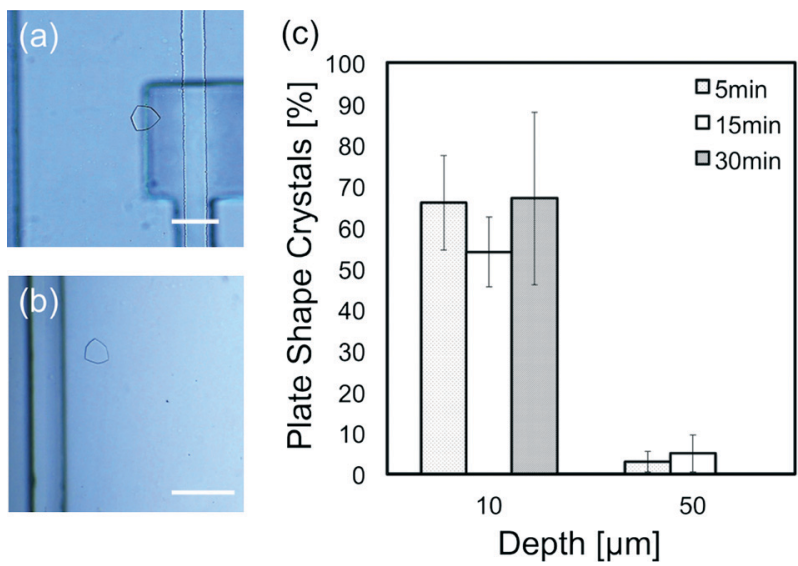

Fig. 5 (a, b) Photographs of the plate-shaped PsGK crystals formed in the microfluidic chip with $10 \mu \mathrm{m}$ deep crystallization chambers. (c) Percentage of the plate-shaped PsGK crystals formed under different mixing times. We observed 150-200 crystallization chambers. The scale bars represent $100 \mu \mathrm{m}$. 
Table 1 Crystallographic data of PsGK crystals prepared using the $10 \mu \mathrm{m}$ depth microfluidic chip method and the hanging drop vapor diffusion method

\begin{tabular}{lll}
\hline Parameter & Chip method $(277 \mathrm{~K})$ & Hanging drop vapor diffusion method $^{a}\left(100 \mathrm{~K}^{2}\right)$ \\
\hline Space group & $P 3_{1} 21$ & $P 3_{1} 21$ \\
Dimensions (̊̊) & $a=b=100.01, c=59.85$ & $a=b=98.61, c=59.73$ \\
Resolution (̊̊) & $50.00-2.84$ & $19.36-1.69$
\end{tabular}

${ }^{a}$ Data for the crystal formed by the hanging drop vapor diffusion method were reported by Oda et al. in the PDB. The PDB ID of PsGK is 3 VPZ.

the diffraction intensity gradually decreased during the X-ray analysis due to crystal degradation. ${ }^{12}$ Although, the X-ray data were enough to resolve the protein structure to the level of amino acid residue side chains, a higher resolution data would be needed to calculate an atomic-level precise $3 \mathrm{D}$ protein structure. Typically, the size of the protein crystal and the flux of the X-ray beam affect the diffracted intensity. The thickness of the PsGK crystal was less than $10 \mu \mathrm{m}$ due to the size limitation of the crystallization chamber. Thus, there were low diffraction intensity crystal planes. Even though this might affect achieving a higher resolution limit, we consider these problems to be addressable by using a high flux synchrotron X-ray beam.

From the results of X-ray analysis, we confirmed that the plate-shaped crystal and the rod-shaped crystal had the same crystallographic parameters. Crystal growth and dissolution of seed crystals were competitive processes. Most of the seed crystals were dissolved because micro seeds were thermodynamically unstable due to their large surface area. When a seed grew to a micrometer sized crystal, a concentration gradient formed around the crystal as shown in Fig. 6. Then, the hexagonal face of the PsGK crystal was oriented parallel to the substrate of the $10 \mu \mathrm{m}$ depth microfluidic chip at the crystal growth stage. In this chip, transport of protein molecules was suppressed compared with the batchwise method and $50 \mu \mathrm{m}$ depth microfluidic chip method. The crystallization environment could change the growth kinetics of each crystal face. Protein crystallization experiments in a special environment, such as microgravity, a magnetic field, or an electric field, have obtained high quality protein crystals and
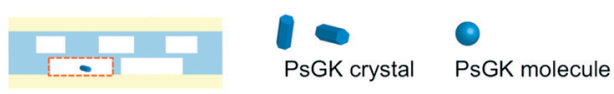

(a) $10 \mu \mathrm{m}$

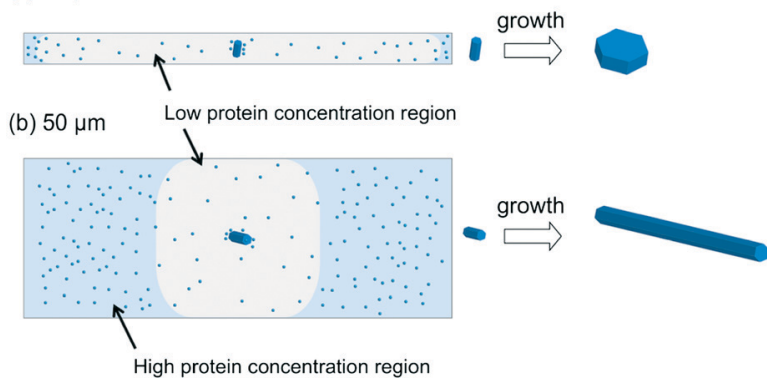

Fig. 6 Schematic illustration of the PsGK crystal growth process in microfluidic chips with crystallization chambers of (a) $10 \mu \mathrm{m}$ and (b) 50 $\mu \mathrm{m}$ depths. affected the protein crystallization behavior. ${ }^{25-27}$ Suppression of the natural convection or the magnetic field orientation effect are expected to be the main factors changing the crystal growth behavior, although a detailed crystal growth mechanism has not been elucidated. Sazaki et al. and Yin et al. also reported that the magnetic field affected the crystal growth rate ratio of each crystal face. ${ }^{28,29}$ Though not protein crystallization, ice crystallization shows a unique crystal growth behavior. ${ }^{30-32}$ Typical hexagonal ice crystals have mainly two crystal faces, the (0001) and the (1010), which correspond to the bottom face and the prismatic face, respectively. The crystallization temperature, namely the supersaturation condition, changes the crystal growth rate of each face. The prismatic face is grown prior to the bottom face at the temperatures from $0{ }^{\circ} \mathrm{C}$ to $-4{ }^{\circ} \mathrm{C}$ and from $-10{ }^{\circ} \mathrm{C}$ to $-20{ }^{\circ} \mathrm{C}$. As a result, the hexagonal plate-shaped ice crystals form. However, the growth rate of the bottom face is faster than that of the prismatic face at low temperature $\left(<-20{ }^{\circ} \mathrm{C}\right)$ and from $-4{ }^{\circ} \mathrm{C}$ to $-10{ }^{\circ} \mathrm{C}$, leading to hexagonal rod-shaped crystals. We consider that the confined crystallization space effected diffusion-controlled protein crystallization and induced the change in growth rate ratio of each crystal face. Consequently, the needle-shaped crystals or the rod-shaped crystals were reformed to the plate-shaped PsGK crystals.

\section{Conclusions}

In conclusion, we have investigated the protein crystal growth behavior in confined space using microfluidic chips with the aim of developing a simple methodology for forming high diffraction quality protein crystals. Our microfluidics-based protein crystallization method was able to control the aggregation of protein crystals without any complicated procedures, external equipment, capping reagents, or a special crystallization environment. Even though aggregated protein crystals such as plate-shaped or needle-shaped crystals were obtained by a conventional batchwise method, our microfluidic chip crystallization method can reform a single protein crystal suitably for X-ray analysis. Moreover, the protein crystal shape can be controlled by the depth of the crystallization chamber. Additional experiments are required to elucidate the protein crystallization behavior in the confined space. We have good expectations that our microfluidic chip protein crystallization approach will become a universal methodology for forming not only high quality protein crystals but also other functional materials. 


\section{Acknowledgements}

We acknowledge K. Matsuura for assistance with the PsGK crystallization experiment. A part of this work was supported by a Grant-in-Aid for JSPS Fellows 26.7016 (to M. Maeki). We thank the beamline staff of the BL07 at the SAGA Light Source for data collection. Some of the X-ray diffraction experiments were performed at the Kyushu Synchrotron Light Research Center Beamline (SAGA-LS/BL07) under Proposal No. $1304026 \mathrm{P}$.

\section{Notes and references}

1 G. Sliwoski, S. Kothiwale, J. Meiler and E. W. Lowe Jr., Pharmacol. Rev., 2014, 66, 334-395.

2 C. S. Tautermann, Bioorg. Med. Chem. Lett., 2014, 24, 4073-4079.

3 W. Liu, A. Ishchenko and V. Cherezov, Nat. Protoc., 2014, 9, 2123-2134.

4 E. Saridakis and N. E. Chayen, Trends Biotechnol., 2013, 31, 515-520.

5 H. Hasenaka, S. Sugiyama, M. Hirose, N. Shimizu, T. Kitatani, Y. Takahashi, H. Adachi, K. Takano, S. Murakami, T. Inoue, Y. Mori and H. Matsumura, J. Cryst. Growth, 2009, 312, 73-78.

6 M. Maeki, H. Yamaguchi, M. Tokeshi and M. Miyazaki, Anal. Sci., 2016, 32, 3-9.

7 L. Li and R. F. Ismagilov, Annu. Rev. Biophys., 2010, 39, 139-158.

8 L. Li, W. Du and R. F. Ismagilov, J. Am. Chem. Soc., 2010, 132, 112-119.

9 B. Zheng, J. D. Tice, L. S. Roach and R. F. Ismagilov, Angew. Chem., Int. Ed., 2004, 43, 2508-2511.

10 C. L. Hansen, E. Skordalakes, J. M. Berger and S. R. Quake, Proc. Natl. Acad. Sci. U. S. A., 2002, 99, 16531-16536.

11 M. Ildefonso, N. Candoni and S. Veesler, Cryst. Growth Des., 2013, 13, 2107-2110.

12 M. Maeki, A. S. Pawate, K. Yamashita, M. Kawamoto, M. Tokeshi, P. J. A. Kenis and M. Miyazaki, Anal. Chem., 2015, 87, 4194-4200.

13 D. S. Khvostichnko, J. M. Schieferstein, A. S. Pawate, P. D. Laible and P. J. A. Kenis, Cryst. Growth Des., 2014, 14, 4886-4890.

14 M. Maeki, S. Yoshizuka, H. Yamaguchi, M. Kawamoto, K. Yamashita, H. Nakamura, M. Miyazaki and H. Maeda, Anal. Sci., 2012, 28, 65-68.
15 M. Maeki, Y. Teshima, S. Yoshizuka, H. Yamaguchi, K. Yamashita and M. Miyazaki, Chem. - Eur. J., 2014, 20, 1049.

16 M. Maeki, H. Yamaguchi, K. Yamashita, H. Nakamura, M. Miyazaki and H. Maeda, Chem. Commun., 2012, 48, 5037-5039.

17 M. Sugishima, H. Sato, Y. Higashimoto, J. Harada, K. Wada, K. Fukuyama and M. Noguchi, Proc. Natl. Acad. Sci. U. S. A., 2014, 111, 2524-2529.

18 T. Yoshida, S. Takahashi and G. Kikuchi, J. Biochem., 1974, 75, 1187-1191.

19 A. S. Pawate, V. Srajer, J. Schieferstein, S. Guha, R. Henning, I. Kosheleva, M. Schmidt, Z. Ren, P. J. A. Kenis and S. L. Perry, Acta Crystallogr., Sect. F: Struct. Biol. Commun., 2015, 71, 823-830.

20 S. Guha, S. L. Perry, A. S. Pawate and P. J. A. Kenis, Sens. Actuators, B, 2012, 174, 1-9.

21 K. Dhouib, C. K. Malek, W. Pfleging, B. Gauthier-Manuel, R. Duffait, G. Thuillier, R. Ferrigno, L. Jacquamet, J. Ohana, J. L. Ferrer, A. Théobald-Dietrich, R. Giegé, B. Lorber and C. Sauter, Lab Chip, 2009, 9, 1412-1421.

22 L. Rong, H. Komatsu and S. Yoda, J. Cryst. Growth, 2002, 235, 489-493.

23 A. McPherson, A. J. Malkin, Y. G. Kuznetsov, S. Koszelak, M. Wells, G. Jenkins, H. Howard and G. Lawson, J. Cryst. Growth, 1999, 196, 572-586.

24 C. E. Kundrot, R. A. Judge, M. L. Pusey and E. H. Snell, Cryst. Growth Des., 2001, 1, 87-99.

25 N. I. Wakayama, M. Ataka and H. Abe, J. Cryst. Growth, 1997, 178, 653-656.

26 T. Sato, Y. Yamada, S. Saijo, T. Hori, R. Hirose, N. Tanaka, G. Sazaki, K. Nakajima, N. Igarashi, M. Tanaka and Y. Matsuura, Acta Crystallogr., Sect. D: Biol. Crystallogr., 2000, 51, 1079-1083.

27 M. Taleb, C. Didierjean, C. Jelsch, J. P. Mangeot, B. Capelle and A. Aubry, J. Cryst. Growth, 1999, 200, 575-582.

28 G. Sazaki, E. Yoshida, H. Komatsu, T. Nakada, S. Miyashita and K. Watanabe, J. Cryst. Growth, 1997, 173, 231-234.

29 D. C. Yin, N. I. Wakayam, H. Wada and W. D. Huang, J. Phys. Chem. B, 2003, 107, 14140-14144.

30 M. Bailey and J. Hallett, J. Atmos. Sci., 2004, 61, 514-544.

31 T. Kobayashi, Phys. Snow Ice, Conf. Proc., 1967, 1, 95-104.

32 V. J. Schaefer, Chem. Rev., 1949, 44, 291-320. 